\title{
Expectations, gains, and losses in the anterior cingulate cortex
}

\author{
Jérôme Sallet, René Quilodran, Marie Rothé, Julien Vezoli, \\ Jean-Paul Joseph, and Emmanuel Procyk \\ Inserm U846, Stem Cell and Brain Research Institute, Bron, France \\ and Université de Lyon 1, Lyon, France
}

\begin{abstract}
The anterior cingulate cortex (ACC) participates in evaluating actions and outcomes. Little is known on how action-reward values are processed in ACC and if the context in which actions are performed influences this processing. In the present article, we report ACC unit activity of monkeys performing two tasks. The first task tested whether the encoding of reward values is context dependent - that is, dependent on the size of the other rewards that are available in the current block of trials. The second task tested whether unexpected events signaling a change in reward are represented. We show that the context created by a block design (i.e., the context of possible alternative rewards) influences the encoding of reward values, even if no decision or choice is required. ACC activity encodes the relative and not absolute expected reward values. Moreover, cingulate activity signals and evaluates when reward expectations are violated by unexpected stimuli, indicating reward gains or losses.
\end{abstract}

Decisions rely on the estimation of the outcome that one will get after a choice is made and an action performed. This estimation, the expected value, is based on the representations of expected and obtained rewardsand more generally, outcomes - that are associated with objects or act through learning (Lee \& Seo, 2007). Value is affected by time of occurrence, probability, frequency, valence, and size of rewards. The ensemble of alternative outcomes available in the current environment can also have an effect on expected values.

The processing of rewards involves a so-called reward system, a network of brain structures including the orbitofrontal cortex (OFC), the ventral striatum, and the mesencephalic dopaminergic system and the anterior cingulate cortex (ACC; Schultz, 2000). OFC activity is modulated by expected outcomes and reflects the relative values assigned by the animals to alternative choices (PadoaSchioppa \& Assad, 2006). Encoding of reward values by dopaminergic neurons is adaptive and relative to the potential outcomes (Tobler, Fiorillo, \& Schultz, 2005). Within the reward system, the ACC has a particular position. Its role most likely consists of building outcome-action associations that serve to elaborate with experience the expected value of particular decisions (Kennerley, Walton, Behrens, Buckley, \& Rushworth, 2006; Rushworth, Walton, Kennerley, \& Bannerman, 2004). There is evidence that the ACC is involved in representing expected values (Amiez, Joseph, \& Procyk, 2006). Thus, one can also expect relative coding of reward values in the ACC.

ACC function also encompasses the voluntary selection of behavior and positive and negative outcome evalua- tions (Amiez, Joseph, \& Procyk, 2005; Shima \& Tanji, 1998; Walton, Devlin, \& Rushworth, 2004). Several theories are proposed to explain ACC activation in humans during tasks that involve active performance monitoring (Holroyd \& Coles, 2002; Kerns et al., 2004; Ridderinkhof, Ullsperger, Crone, \& Nieuwenhuis, 2004; Rushworth et al., 2004). Signals from ACC have been interpreted in terms of the detection of conflict in processing competitive representations - for example, alternative action plans (Botvinick, Cohen, \& Carter, 2004). These ACC signals could also possibly reflect detection of events at odds with expectations, especially in terms of rewards (Gehring \& Willoughby, 2002; Holroyd \& Coles, 2002; Rushworth et al., 2004). Indeed, ACC feedback-related activity varies with reward prediction errors during learning (Amiez et al., 2005; Holroyd \& Coles, 2002; Matsumoto, Matsumoto, Abe, \& Tanaka, 2007).

The present experiments were designed to further investigate the representations of expected values. We report recordings in the ACC of monkeys performing two tasks: the scaled-reward task, and the expect task that involved neither making a decision between alternative choices nor learning particular reward values. The first task was used to evaluate whether modulations of ACC activity by values would be influenced by context - that is, by the other rewards available within a block of trials and by the status of reward amount (fixed vs. probabilistic). The second task was designed to search for ACC activity related to the evaluation of events signaling a change in outcome that would be at odds (with gain or loss) with the present expectation.

J. Sallet, sallet@lyon.inserm.fr 
A

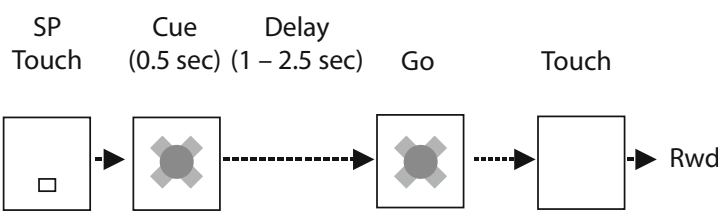

B

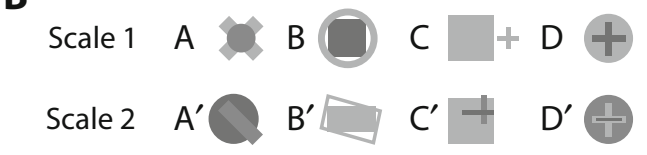

C

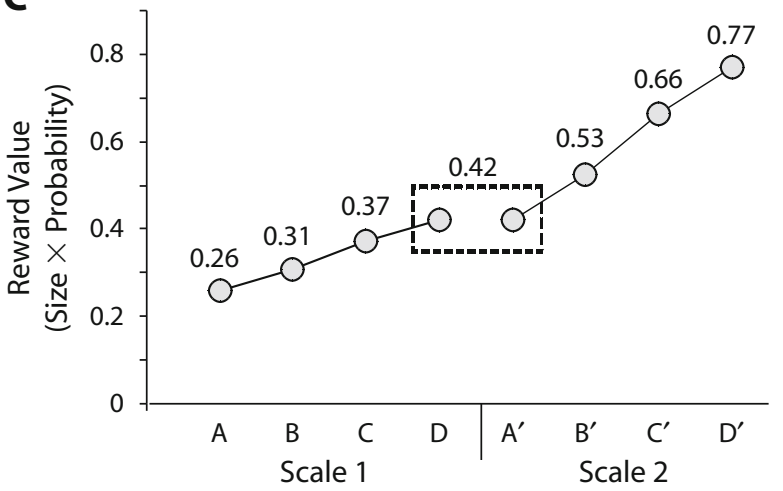

D

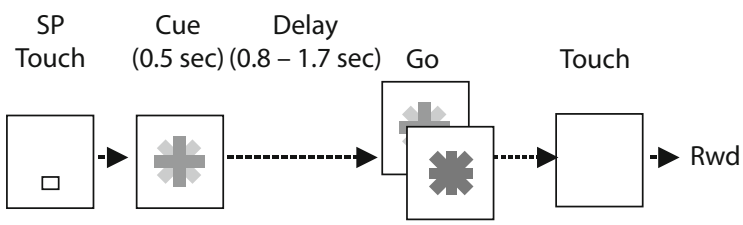

E

Expected

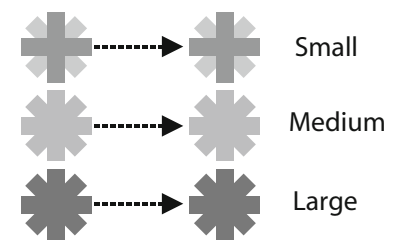

Unexpected

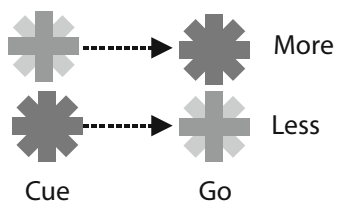

Figure 1. Behavioral tasks (A-C, scaled-reward task, D-E, expect task). (A) Schematic representation of the scaled-reward task. The animal started a trial by touching the starting position (SP) and maintaining the touch. Then a cue switched on for 0.5 sec. The cue indicated the amount of reward delivered in case of successful trial. After a variable delay, the item switched on again (go signal), and the animal could release the starting position and touch the item. Six hundred milliseconds after the touch, the animal received the reward. (B) Cues used for the four reward conditions in each scale. Cues from one scale were presented in a block of trials. (C) Graphic showing the reward value (size $\times$ probability) associated with each reward condition in each scale. (D) Schematic representation of the expect task. The task is similar to the reward-scaled task that was described in A. The major difference is that in some trials, the go item is different from the cue (in about $25 \%$ of trials). (E) Representation of the cue/go pairs used in this study. In three conditions, the go is identical to the cue, and the expected reward is delivered. In two conditions, the go item is different from the cue, and the reward to be obtained is larger (more) or smaller (less) than the expected reward.

\section{METHOD}

\section{Behavior}

Housing, surgical, electrophysiological, and histological procedures were carried out according to the European Community Council Directive (1986), the Ministère de l'Agriculture et de la Forêt, the Commission Nationale de l'Expérimentation Animale, and the Direction Départementale des Services Vétérinaires (Lyon, France). Each animal was seated in a primate chair within arm's reach of a tangent touch screen (Microtouch System) coupled to a TV monitor. In the front panel of the chair, an arm-projection window was opened, allowing the monkey to touch the screen with one hand. A computer recorded the position and accuracy of each touch. It also controlled the presentation via the monitor of visual stimuli (color shapes), which served as light targets (CORTEX software, NIMH Laboratory of Neuropsychology, Bethesda, MD). Eye movements were monitored using an Iscan infrared system (Iscan Inc.).

Two male rhesus monkeys were trained in both the scaled-reward task and expect task (Figure 1). In each task, the stimuli were fixed from one day to another. They were presented in the center of the screen, were $4 \times 4 \mathrm{~cm}$ in dimension, and were composed of superimposed shapes of different colors. In both tasks, each trial was selfinitiated by the monkey upon touching a starting position (SP) on the screen. The animal then had to hold this position. After a short delay, a visual cue appeared at the center of the screen for $500 \mathrm{msec}$. After a waiting delay (from 1,000 to $2,500 \mathrm{msec}$ in the scaled-reward task and from 800 to $1,700 \mathrm{msec}$ in the expect task), a go signal appeared. The animal had to touch the stimulus that was given at the go signal. A reward juice was delivered $600 \mathrm{msec}$ after the touch.

\section{Scaled-Reward Task}

The scaled-reward task was designed to measure the effect of reward size expectation on ACC activity and to test whether expected reward sizes were encoded in a linear or relative fashion by the firing rates. Moreover, different scales of reward were presented in different blocks in order to test the effect of context (i.e., the other potential rewards in a block). Two scales of four stimuli-reward couples were used. In each scale, the extremes were associated with fixed reward, and the mediums were associated with probabilistic rewards. Reward values for each stimulus were calculated as follows: value $=$ size $(\mathrm{ml}) \times$ probability of the reward.

In the lower scale (Scale 1), the stimuli were named A, B, C, and D. In the larger scale (Scale 2), the stimuli were named $A^{\prime}, B^{\prime}, C^{\prime}$, and $D^{\prime}$. Reward value associated with each stimulus is reported in Figure 1.

The different values are listed below.

Scale 1: A, $0.26=0.26 \mathrm{ml} \times 1 ; \mathrm{B}, 0.31=(0.26 \times 0.7)+(0.42 \times$ $0.3) ; \mathrm{C}, 0.37=(0.26 \times 0.3)+(0.42 \times 0.7) ;$ and $\mathrm{D}, 0.42=0.42 \times 1$.

Scale $2: \mathrm{A}^{\prime}, 0.42=0.42 \times 1 ; \mathrm{B}^{\prime}, 0.53=(0.42 \times 0.7)+$ $(0.77 \times 0.3) ; \mathrm{C}^{\prime}, 0.66=(0.42 \times 0.3)+(0.77 \times 0.7) ; \mathrm{D}^{\prime}, 0.77=$ $0.77 \times 1$. 
Importantly, in order to test the effect of context, the reward value of $\mathrm{A}^{\prime}$ was equal to that for $\mathrm{D}$, with the difference that $\mathrm{A}^{\prime}$ is the minimum reward of Scale 2 and $D$ is the maximum reward of Scale 1.

Scales were presented in blocks of trials that were usually interspaced by either rest or a block of trials of the expect task. Thus, the time interval between tests could vary from 1 to $15 \mathrm{~min}$. Each scale was presented once or twice, depending on the stability of recordings.

\section{Expect Task}

The expect task was designed to test whether ACC generates specific signals at the onset of events that indicate a discrepancy between the expected reward and the reward to be obtained.

In three conditions, three stimuli were associated with small $(0.21 \mathrm{ml})$, medium $(0.48 \mathrm{ml})$, or large $(0.96 \mathrm{ml})$ rewards. In about $75 \%$ of small and large trials and in $100 \%$ of medium trials, the go signal was identical to the cue, thus confirming the expected reward. In $25 \%$ of both small and large trials, the go signal did not match the cue. After a cue indicating a small reward, the item referring to a large reward appeared and indicated more (than what was expected). After a cue indicating a large reward, the item referring to a small reward appeared, indicating less (than what was expected). There were five different types of trials: large-large, medium-medium, small-small, large-less, and small-more. The procedure for mixing conditions was made by providing in the random selection additional trials for incongruent conditions. Thus, at the beginning of trials, the average probabilities for the cues small, medium, and large to appear were $.37, .27$, and .36 , respectively (computed from 1,687 trials).

\section{Behavioral Measures}

Monkeys were required to respond within defined time limits for reaction time (RT) and movement times. We tested various time limits from a 300 to $600 \mathrm{msec}$ delay for both RT and movement time. These constraints were adapted online to allow the animal to perform at an appropriate performance level in order to give sufficient numbers of rewards. The RT for arm movements (from go to start-position release) and the number of errors were measured. Errors of several types (no start, no go, late response, early response) were analyzed separately.

\section{Recordings}

Monkeys were implanted with a head-restraining device, and an atlas-guided (Paxinos) craniotomy was performed in order to expose an aperture over the prefrontal cortex. A recording chamber was implanted with its center placed at stereotaxic anterior level +31 . A vertical chamber implanted vertically on the midline was used for Monkey P. A lateral chamber positioned at $32^{\circ}$ from the vertical plane was used for Monkey M. Neuronal activity was recorded using epoxycoated tungsten electrodes (1-4 M $\Omega$ at $1 \mathrm{kHz}$; FHC Inc., USA). One to four microelectrodes were placed in stainless steel guide tubes and independently advanced into the cortex through a set of micromotors (Alpha-Omega Engineering, Israel). Neuronal activity was sampled at $13 \mathrm{kHz}$ resolution. Electrode tracks and recording locations were verified using anatomical MRI for Monkey P and histology for Monkey M. Recording sites covered an area extending over about $6 \mathrm{~mm}$ (anterior to posterior), in the dorsal bank of the anterior cingulate sulcus, at stereotaxic antero-posterior levels superior to $\mathrm{A}+30$, and at depths superior to $4.5 \mathrm{~mm}$ from cortical surface. The recording zone corresponds to a region that has been recorded in previous reports and in which error-related activity has been observed (Amiez et al., 2006; Ito, Stuphorn, Brown, \& Schall 2003; Procyk, Tanaka, $\&$ Joseph, 2000). This part of the anterior cingulate cortex lies at the same anterior level as the SEF, and includes part and goes anterior to the rostral cingulate motor area (CMAr), as evaluated from previous publications (Ito et al., 2003; Shima et al., 1991).

Single unit activity was identified using online spike sorting (MSD, AlphaOmega). The activity of single neurons was analyzed with respect to different events and outcomes (conditions) by using averaged peristimulus histograms (PSTHs) and trial-by-trial spike counts. Statistical evaluations of neural activity were done using average spike frequency measured in separated time epochs delineated in each trial (NeuroExplorer, Nex Technology; MatLab, The MathWorks Inc.). PSTHs had a binning of $0.01 \mathrm{sec}$ and were Boxcar averaged. Average population activity was smoothed using a lowess fitting (precision 0.02; Statistica, Statsoft Inc.).

\section{Analyses}

For the scaled-reward task, we selected 7 epochs: SP, onset starting position to $300 \mathrm{msec}$ after touch of the position; cue, $500 \mathrm{msec}$ period following cue onset; delay, the first $500 \mathrm{msec}$ of the delay; go, from the go signal to the starting position release; movement, from the release lever to target touch; post touch, from target touch to reward delivery, and reward, $600 \mathrm{msec}$ following reward delivery. A baseline epoch was defined as the last $500 \mathrm{msec}$ of the ITI. Expected reward-size effects were tested using a two-way ANOVA, with epoch $\times$ reward as factors $(p<.05)$.

One issue is whether the neural activity encodes expected reward values monotonically. In order to address this problem, we first selected one particular epoch per cell. We used a selectivity index based on a nonparametric one-way ANOVA (Kruskal-Wallis test, the factor reward size). The analysis extracted a $\chi^{2}$ value. We used it as a measure of the variance explained by reward size. The epoch in each cell with the largest $\chi^{2}$ value was selected for further analyses. A normalization was then adopted that (1) transformed activity in Trial A $\left(\mathrm{A}^{\prime}\right)$ and Trial $\mathrm{D}\left(\mathrm{D}^{\prime}\right)$ to 0 and 100 , respectively, and (2) linearly adjusted the value observed in the other trials to this new scale. Let $X$ be the average activity measured in 1 epoch; we normalized $\mathrm{X}$ by: $\mathrm{X} \rightarrow$ $100 \times(\mathrm{X}-\mathrm{A}) /(\mathrm{D}-\mathrm{A})$, where $\mathrm{A}$ and $\mathrm{D}$ represent the average activity for the same epoch in Trials A and D. With this transformation, the activity in both Trial A and Trial D in particular became equal to 0 and 100, respectively, independently of whether activity in Trial D was initially larger or smaller than that in Trial A (see Figure 5). The normalization was applied using activity for Trials A and D as reference for neurons having significantly different activity in Trials A and $\mathrm{D}$. Activity in $\mathrm{A}^{\prime}$ and $\mathrm{D}^{\prime}$ were used as references for neurons having significantly different activity in trials $\mathrm{A}^{\prime}$ and $\mathrm{D}^{\prime}$.

For the expect task, change in neural activity (neural response) was tested for being event related. A change in activity was considered to be significant if in at least one condition, the average activity measured within the 500 -msec post-event (post-cue or post-go) exceeded more than twofold the standard deviation of the preevent average activity (taken during the window $-600 /-200$ msec preceding the event alignment time), and remained above this threshold for more than six $0.01 \mathrm{sec}$ bins. Average activity was measured by detecting the peak in average activity (all conditions combined) in the $500 \mathrm{msec}$ following event times and computing the average activity in a -160 -msec to $\mathrm{a}+160$-msec window around the peak time. The average activity was standardized to the mean and standard deviation of the baseline activity taken from -600 to $-200 \mathrm{msec}$ before event onset. Resulting data were expressed in number of sigma $(S D)$ of the baseline.

Average population activity on single units showing significant effects was constructed to illustrate the statistical results. This format was also used to observe the dynamic of the average activity in the different subpopulations.

\section{RESULTS}

\section{Scaled-Reward Task: Expected Values and ACC Activity}

\section{Behavior}

Errors in Monkey P (Figure 2A) were primarily due to no-go responses (no arm movement toward the target) and to a small proportion of no-start responses (trial not initialized). Error rates tended to be smaller for large rewards within each scale. RTs were statistically the same in all trials. In Monkey M (Figure 2B), the rates of errors (because of no-go trials) were smaller than those in Monkey P. They 
also tended to be smaller for large rewards. RTs did not vary within scales, only between scales. A global modulation of error rate by reward-size expectation in Scale 1 (assessed by the ANOVA; $p<.05$ ) was observed for both monkeys. Furthermore, for both monkeys, we observed a significant difference in performance (error rate or RTs) between $\mathrm{D}$ trials and $\mathrm{A}^{\prime}$ trials, indicating a context effect (Figures 2A and 2B).

In summary, the performance of the two monkeys was modulated by the expected size of rewards, although the context effect (D vs. $A^{\prime}$ ) and the block effect on errors were different between the two monkeys.

\section{Neuronal Data}

This scaled-reward task was designed to assess how ACC neurons encode reward values. Our a priori hypothesis was that ACC encodes relative reward values in a context-dependent manner. Thus, the relative coding of rewards in one definite scale should be similar to those in another scale, even if absolute reward values are different.

We first evaluated whether the neural activity recorded for each cell was modulated by the size of expected rewards. Figure $3 \mathrm{~A}$ shows the activity of a typical example cell. A two-way ANOVA (epoch $\times$ reward value, threshold $p<.05$ ) was performed independently for the two scales. Out of 62 cells, recorded for at least one reward scale (60 cells recorded for Scale 1, the smaller rewards, and 57 for Scale 2, the larger rewards), 24 and 25 showed a main effect of reward value and/or an interaction between reward value and epoch. Thus, the activity of $40 \%$ and $45 \%$ of ACC cells was modulated by expected reward values.

In order to characterize the type of coding (linear, tuned, etc.) that was performed by the cells, we worked on a group of cells $(n=23)$ that fulfilled the following requirements: (1) They were recorded in both scales; (2) they revealed an effect of reward value in at least one scale (ANOVA, see above); and (3) their baselines for Scales 1 and 2 were not significantly different (Wilcoxon rank sum test, $p<.05$ ). We then isolated the epoch for which the reward index was maximum (see Method). The reward index indicates the strength of the modulation by reward value. On average, maximum indices were found at epochs surrounding arm movements, suggesting that the best discrimination of reward values was taking place in association with movement planning and execution (Figure 4).
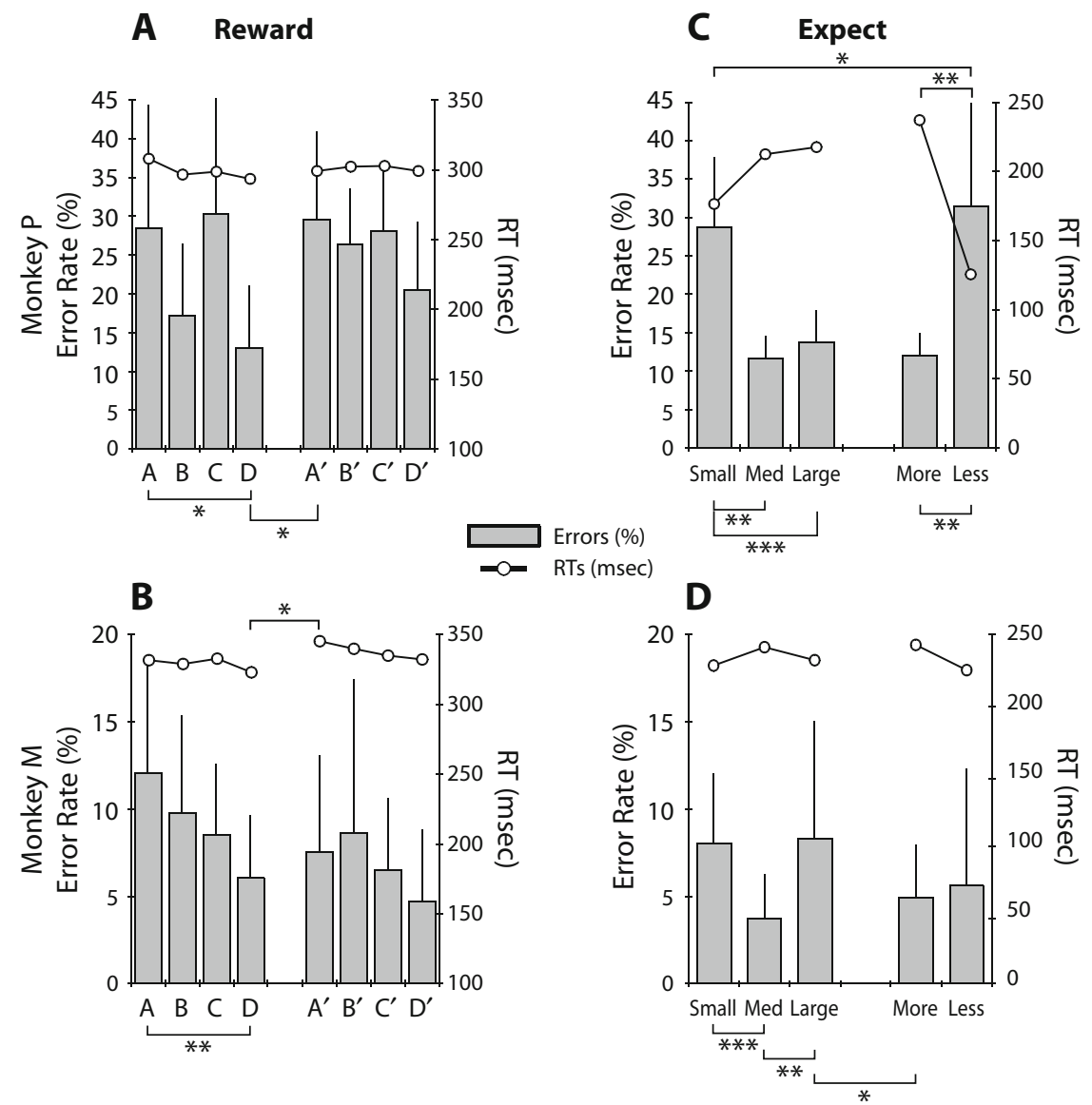

Figure 2. Behavioral data for Monkeys $P$ and $M$ in the scaled-reward (A-B) and expect tasks (C-D). Error rates (bars) and reaction times (RT; plots). One-way ANOVA: ${ }^{*} p<.05$. ${ }^{* *} p<.01 .{ }^{* * *} p<.001$. Significance of tests are shown on top for RT and below histograms for errors. 
A
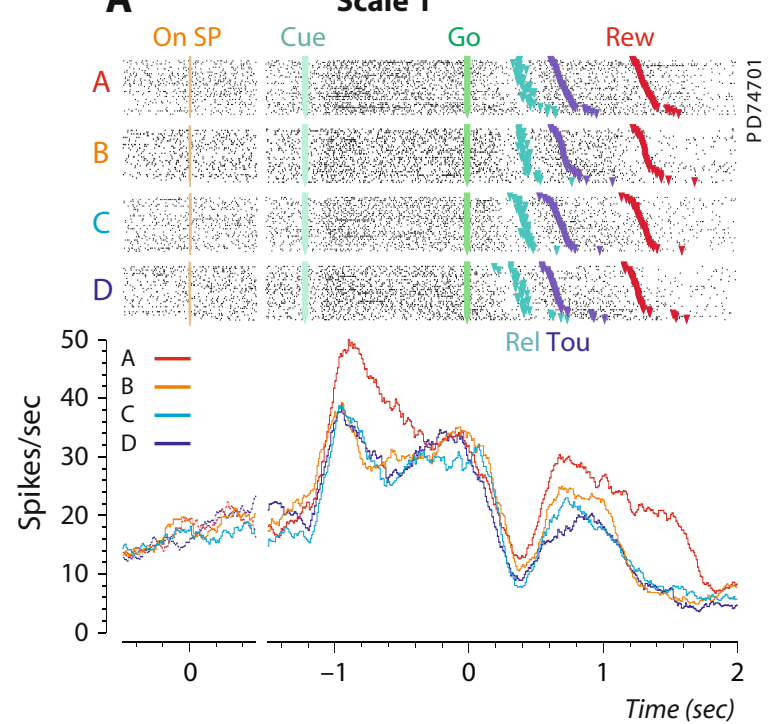

Scale 2
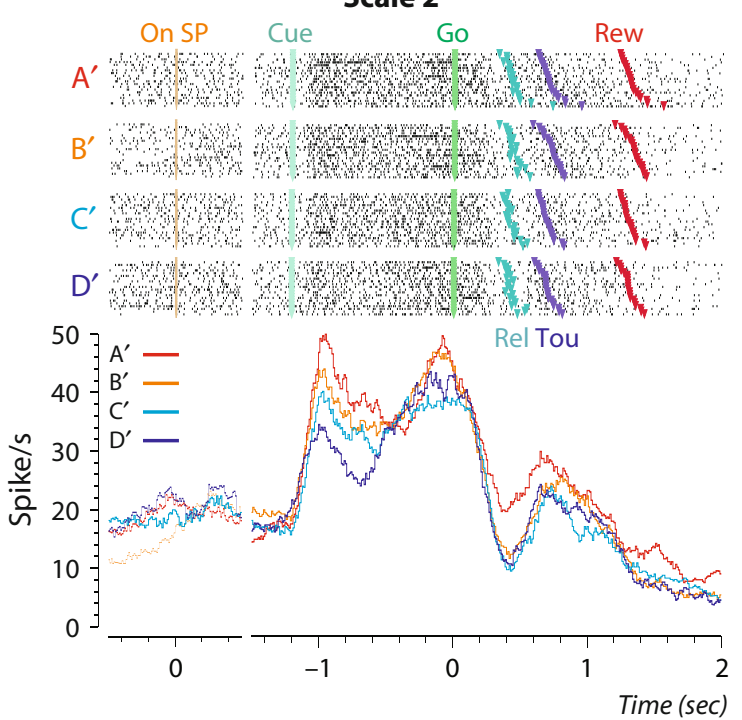
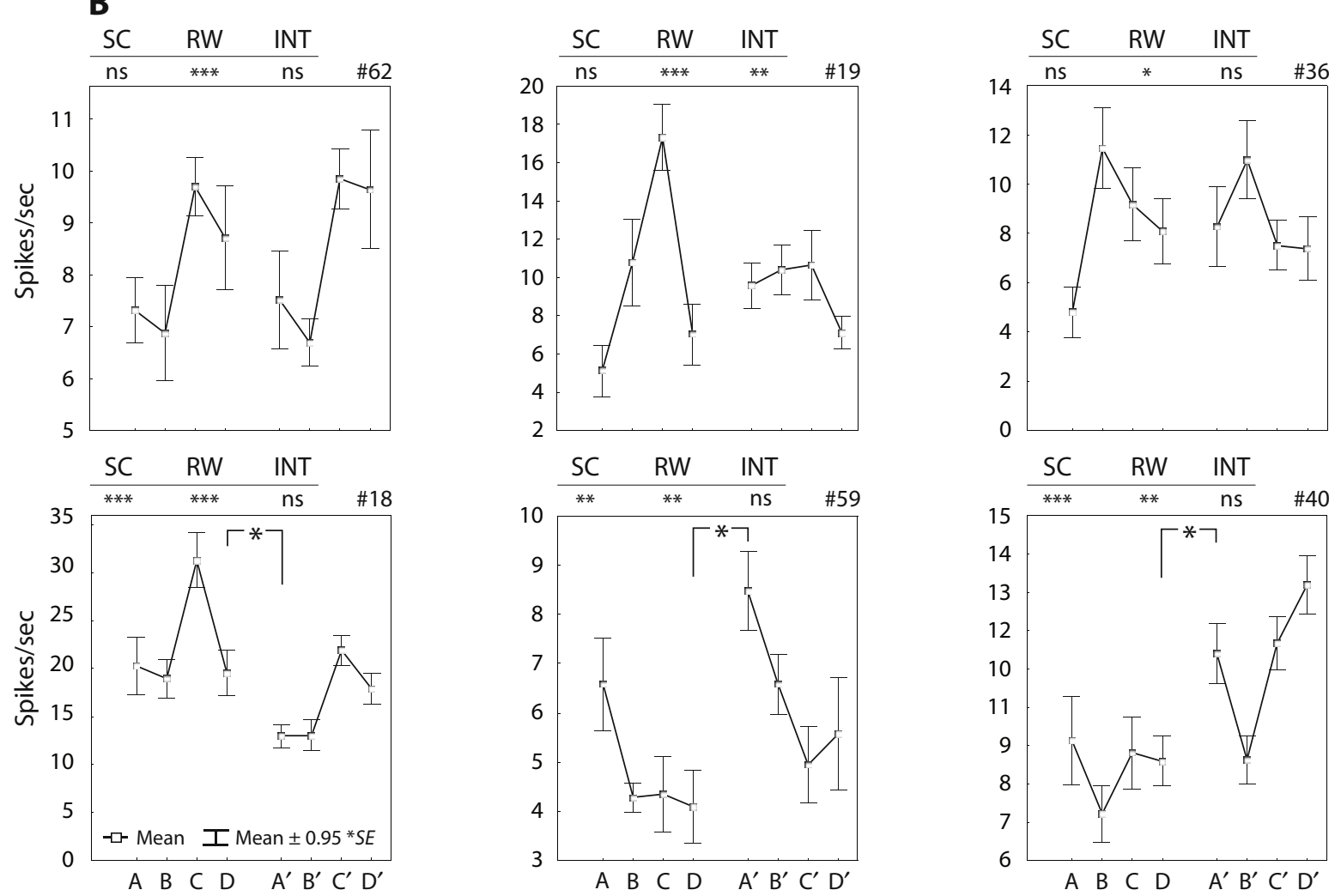

Figure 3. Neuronal data in the scaled-reward task. (A) Activity of one neuron that varies with reward values. Rasters and histograms are presented for Scale 1 (left) and Scale 2 (right) for each reward value (colors). Activity is aligned on the onset of the starting position (On SP), the cue onset (Cue), and on the go signal (Go). Colored triangles in rasters indicate the release of the starting position (Rel), the touch of the target (Tou), and the reward delivery (Rew). (B) Average activity for six individual cells. Each panel represent the eight averages corresponding to the reward values of the two scales $\left(A, B, C, D\right.$ and $A^{\prime}, B^{\prime}, C^{\prime}, D^{\prime}$, respectively). Statistical differences between average activity in $\mathrm{D}$ and $\mathrm{A}^{\prime}$ are indicated inside the plot (Wilcoxon; $\left.{ }^{*} p<.05\right)$. Above each panel, the results from an ANOVA [scale $(d f=1) \times \mathrm{RW}(d f=3)$ ] testing the effect of scale and relative reward values. The significance of each factor and of interactions are indicated $\left({ }^{*} p<.05 ;{ }^{* *} p<.01\right.$; $\left.{ }^{* * *} p<.001\right)$. Note the similarity between the distributions of averages found in the two scales.

For the 23 cells, we plotted the average activity measured in the selected epoch. Six examples are presented in Figure 3B. Various relationships between expected re- ward values and neural activity were found. The preferred expected value (the one for which maximum activity was found) within a scale could be the smallest (A or $\mathrm{A}^{\prime}$ ), the 


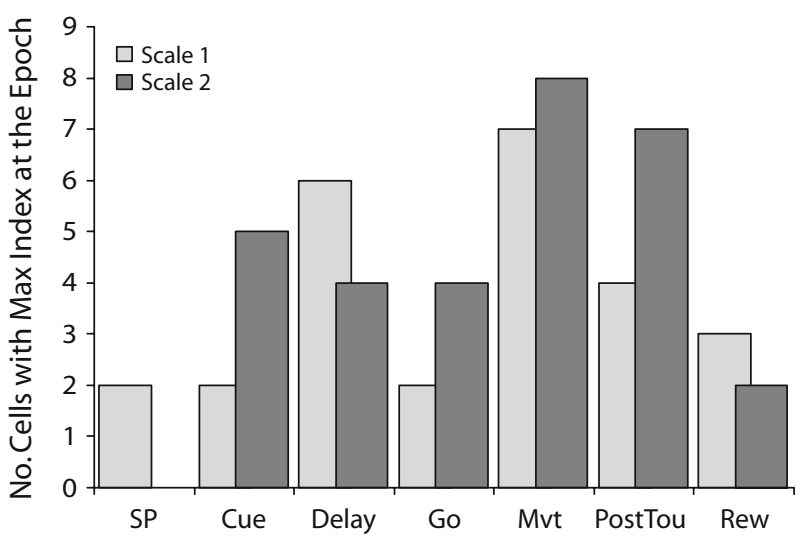

Figure 4. Scaled-reward task. Distribution of epochs with maximum reward index (revealed by a $\chi^{2}$ ). Data are given for Scales 1 and 2 separately.

largest $\left(\mathrm{D}\right.$ or $\left.\mathrm{D}^{\prime}\right)$, or one of the intermediate $\left(\mathrm{B}, \mathrm{C}, \mathrm{B}^{\prime}\right.$, or $\left.\mathrm{C}^{\prime}\right)$. The encoding of expected reward sizes was different from cell to cell, as would be expected if cells had different tunings.

The experiment was designed specifically to test whether encoding of expected reward values in one definite scale is similar in another scale, even if absolute reward values are different (relative encoding).

We first tested whether scale and reward were two interacting factors. A two-way ANOVA $(p<.05$; scale $\times$ reward-rank. For reward-rank, ranks are 1, 2, 3, and 4 for $\mathrm{A}, \mathrm{B}, \mathrm{C}$, and $\mathrm{D}$ and for $\mathrm{A}^{\prime}, \mathrm{B}^{\prime}, \mathrm{C}^{\prime}$, and $\mathrm{D}^{\prime}$, respectively) was performed on the epochs with maximum index. Among the 14 out of 23 cells showed a reward-rank effect (at $p<.05$ ), only 4 showed an interaction with the factor scale. Statistical results are shown in Figure 3B for the examples. The absence of interaction in 10 out of 14 cells (two thirds) reveals the cases for which there was no change in relative encoding between the scales. Thus, a majority of the analyzed cells showed a relative and adaptive coding of expected rewards.

In order to further compare the two scales, we selected the cells for which the average activity differed between the extreme rewards (A vs. D and/or A' vs. D'). A Wilcoxon rank-sum test (at $p<.05)$ performed on epochs presenting the maximum index of selectivity revealed 11 cells with significant differences between $\mathrm{A}$ and $\mathrm{D}$ and/or between $\mathrm{A}^{\prime}$ and $\mathrm{D}^{\prime}$ (one cell showed an effect for both tests). Average population data for the 11 cells are shown in Figure 2. The left panel shows the average responses for six neurons with significantly different activity for A and D. For each neuron, the four averages of activity recorded in each scale (eight values) has been normalized so that activity for A is at 0 and activity for D is at 100 (see Method). Data are plotted against absolute reward values on the abscissa. The right panel shows the average responses for the other six cells with significantly different activity for $\mathrm{A}^{\prime}$ and $\mathrm{D}^{\prime}$ (one cell is also contributing to the graph in the left panel). For these cells, the normalization of the activity is based on the average activity for $\mathrm{A}^{\prime}$ and $\mathrm{D}^{\prime}$.

The two plots in Figure 5 confirm at the population level that there is no monotonic increase or decrease according to the absolute reward values from one scale to another. Although the differences between rewards in one scale tend to disappear in the other, the averages stay in the same range. The graph shows as a comparison the activity that would be expected if it were linearly coding for the relative reward values within a scale (dashed lines).

In summary, $45 \%$ of cells were modulated by expected reward sizes. However, the subpopulation of cells that we
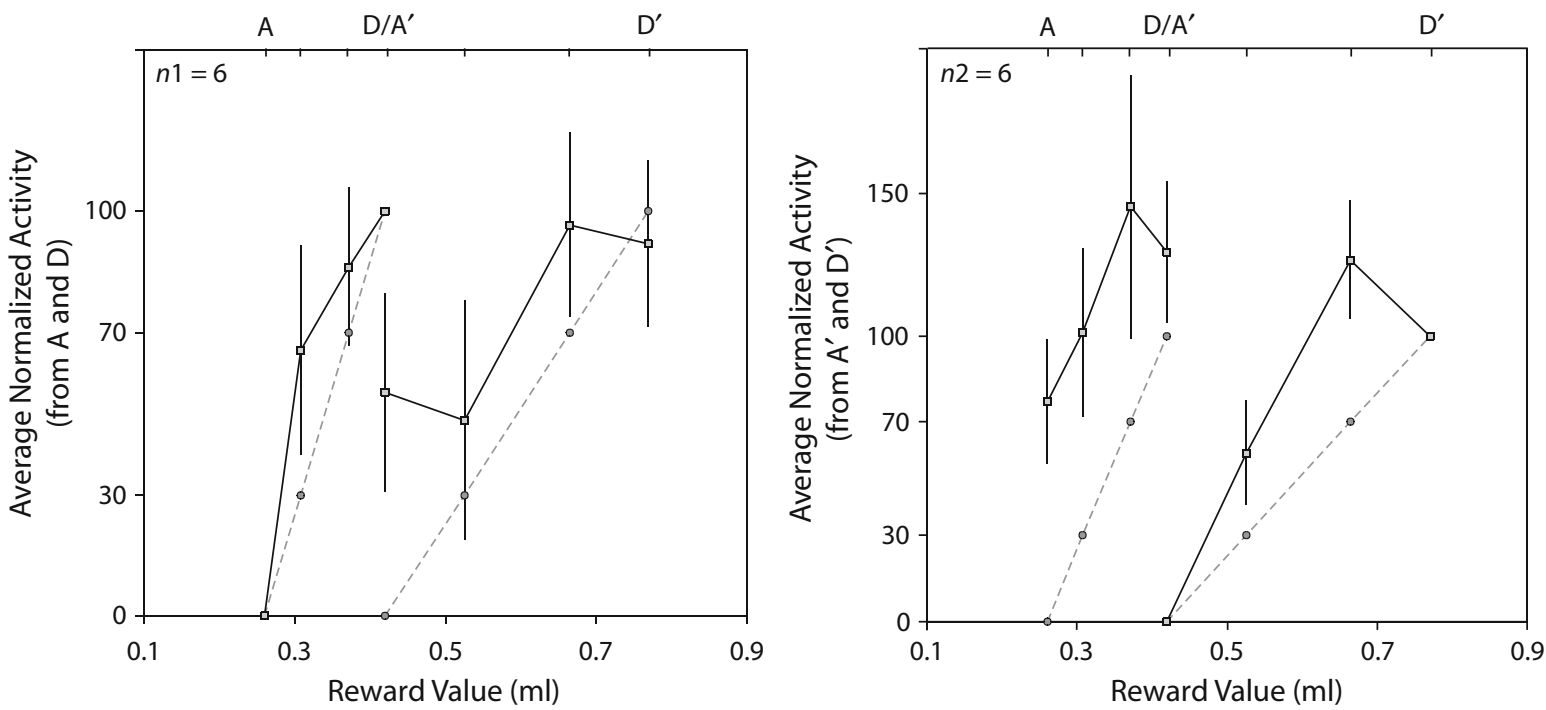

Figure 5. Average normalized activity in the two scales for 11 cells with different activity in $A$ and $D($ left; $n 1=6)$ and/or in $\mathrm{A}^{\prime}$ and $\mathrm{D}^{\prime}$ (right; $n 2=6$ ). In each panel, the average activity for each reward value is normalized according to either the values in $A$ and $D$ (left) or $A^{\prime}$ and $D^{\prime}$ (right). The abscissa is in milliliters, and the corresponding reward value names are displayed on the top. $D$ and $A^{\prime}$ have the same value. The graph shows as a comparison the activity that would be expected if it were linearly coding for the relative reward values within a scale (dashed lines). 
analyzed showed that the encoding of expected reward sizes available in one block is relative and not absolute.

\section{Expect Task: Unexpected Gains or Losses and ACC Activity}

\section{Behavior}

In Monkey P (Figure 2C), errors were the result of no-go responses. The proportion of no-go trials was larger for small rewards (small and less trials). The error rate was also higher in less conditions than in more conditions. RTs were shorter for small rewards. Note that the proportion of no-go errors was quite large and created problems for getting sufficient data in the less condition.

Monkey M (Figure 2D) made fewer no-go trials. Error rates and RTs were similar for small and large rewards, and for more and less conditions. Significant differences were observed in error rates only in small versus medium, medium versus large, and large versus more conditions.

In summary, the effects of reward schedules (reward size and more vs. less) were similar in the two monkeys, but were overall weaker for Monkey M.

\section{Neuronal Data}

A total of 95 cells (59 in Monkey M and 36 in Monkey P) were recorded long enough to study activity at the go signal in correctly performed congruent and incongruent trials (trials in which the monkey received a reward).

For 42 neurons $(44 \%)$, the activity significantly increased within $500 \mathrm{msec}$ after the onset of at least one of the three cues ( $>2 S D$ s from precue; see Method). Of these cue-related activities, $45 \%(n=19)$ showed an effect of cue identity/expected reward size (ANOVA $p<.05$ ).

Out of the 95 cells, $56(59 \%)$ showed significant gorelated activity (a significant increase) $500 \mathrm{msec}$ after the go signal in at least one of the five conditions (less, more, small, medium, and large) ( $>2 S D$ s from pre-go; see Method).

We were especially interested in whether cells could show distinct activity for more and/or less conditions after the go signal. A total of 16 cells had significant $(>2 S D$ s) and maximum average activity at the go signal for the more condition, in comparison with the small, medium, less, and large conditions. Eighteen other cells had significant and maximum average activity for the less condition in comparison with the small, more, medium, and large conditions. Two examples are shown in Figure 6. Figure $6 \mathrm{~A}$ illustrates the phenomenon for more conditions. In the small-more condition, the more item (which is identical to the large item) induces higher activity in this cell than in all other conditions. There is no particular dis-

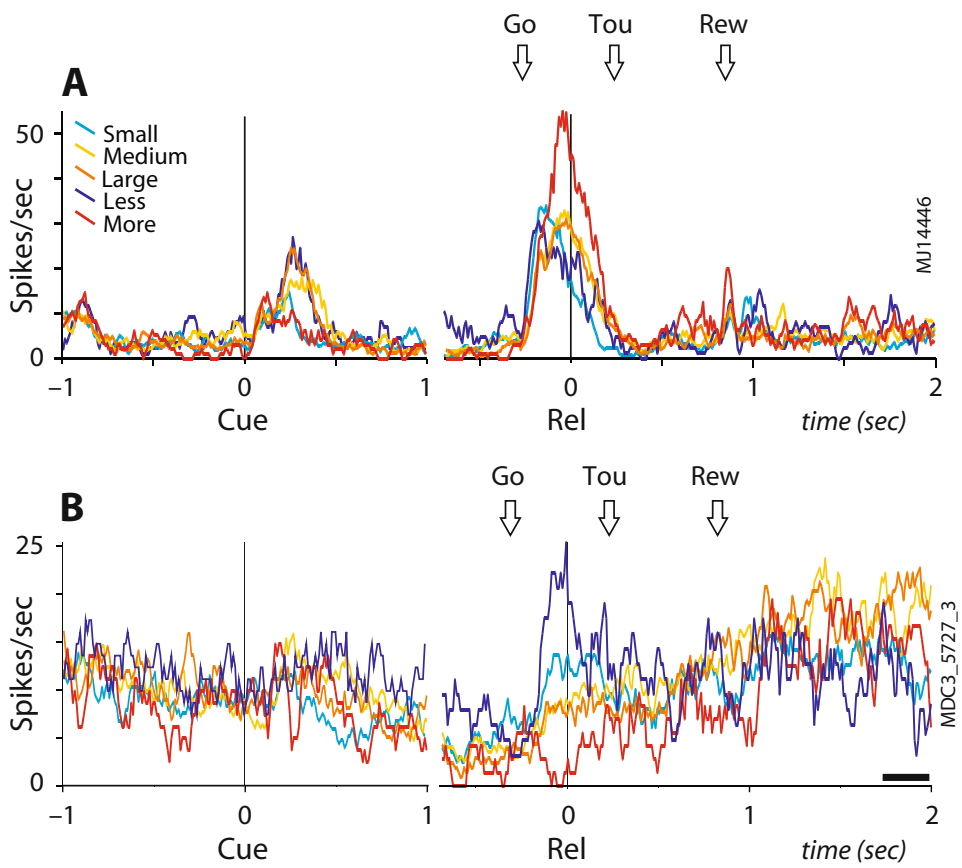

Figure 6. Examples of neurons in the expect task. Two examples of unit activities recorded in the five conditions. The histograms are aligned on the cue (Cue) on the left side of the figure, and on the release of the starting position (Rel), on the right side. Colored lines correspond to the different conditions (large, dark, orange dark; medium, light orange; small, blue; more, red, and less, dark blue). The cell in (A) shows an increase of activity at the cue that is statistically larger for cues announcing larger rewards than small rewards (small and more). The cell is more active at the go signal for the more condition. The cell in (B) presents a maximum activity at the go signal for less, and lower activity for the other conditions. 
charge to the unexpected small. A similar effect is found for less neurons (see a single example in Figure 6B). The more and less effects are observed in the two population activity (Figures 7A and 7C). These data reveal that the ACC is signaling the discrepancy between the expected reward and the reward announced at the time of the go signal, even before any reward is delivered.

Modulation of activity at the cue and at the different go signals could possibly reflect a pure effect of probability of occurrence. The more item has a probability of .25 to appear after a cue indicating a small reward; the large cue has a probability of .36 of being presented, and the expected large go has a probability of .75. In this hypothesis, the activity should increase or decrease with probabilities This was not the case for both less and more cells (Figures 7B and 7D). This result suggests that the more and less signals reflect the unexpected gain or loss in reward.

An important question concerns the specificity of ACC responses, whether they are always coding in one direction (more or less) or whether there are other activities reacting to both incongruent conditions (more and less) without specifying gain or loss. No cell was found to have significant activity in both more and less conditions, in comparison with the small and large conditions that would have revealed cells signaling a pure incongruent go signal.

\section{DISCUSSION}

Our data show that ACC activity encodes expected reward values and that the coding is relative to the context. In our protocol, the context was the ensemble of alternative reward values given in the current block of trials. In addition, ACC unit activity signals the violation of expectations in terms of gain or loss of reward.

\section{Limitations}

The effects of reward-size expectation or of incongruent conditions on behavioral performances were presentalthough not strictly identical - for the two animals. It is possible that the online adjustments of time constraints might have slightly influenced the speed and accuracy of responses for each animal. This procedure was used to adapt to each animal and avoid no-response or lateresponse errors that prevented us from recording neuronal activity for a sufficient number of trials. At the same time, it forced the animal to attend to the go signal. Other procedures might be considered to avoid behavioral discrepancies between subjects. In any case, the effects of experimental conditions on neuronal activity in the two monkeys were comparable.

The number of task-related neurons used to compare activity in Scales 1 and 2 in the scaled-reward task is low. Criteria on trial numbers also induced important data selection for the expect task for which there was a limited number of correct trials in the less condition. Conservative criteria were defined in order to avoid experimental biases. These criteria increased the relevance of our statistical results.

For both tasks, we used behavioral situations in which there was no challenge in terms of response choice. Doing so might have led us to record ACC at its minimal in- volvement. Indeed, several lines of evidence suggest that ACC is much involved in situations in which actions are self-selected, outcomes are uncertain, and high behavioral flexibility is required (Procyk, Amiez, Quilodran, \& Joseph, in press; Rushworth, Behrens, Rudebeck, \& Walton, 2007; Rushworth et al., 2004). Yet, the recorded task-related activities showed different coherent effects of context and reward-size expectations in the two tasks.

\section{Expectations of Rewards}

Amiez et al. (2006) have shown that in a probabilistic learning task in which decisions are adapted to maximize rewards, ACC neurons code for the average reward value of the best choice, the task-value (Amiez et al., 2006). Presently, we show that ACC activity is modulated by expected reward sizes even in a simple task that involved neither decision between several alternative choices nor learning of action values.

A near monotonic encoding of reward quantities within scales could be inferred from cells in which the response to $\mathrm{D}$ is different from the response to $\mathrm{A}$, or the response to $\mathrm{D}^{\prime}$ is different from the response to $\mathrm{A}^{\prime}$ (Figure 5). For a subpopulation of cells, the activity in anticipation of fixed rewards was overall larger or smaller than that for intermediate rewards ( $\mathrm{D}$ and $\mathrm{C}, \mathrm{D}^{\prime}$ and $\mathrm{C}^{\prime}$ ). Interpretation of these responses is not straightforward. It may reflect the different reward schedules (certain vs. probabilistic). A monotonic pattern of value coding in ACC has been observed previously in a choice task (Amiez et al., 2006). A monotonic coding of reward values might be a prerequisite to make optimal decisions in probabilistic environments. Thus, it might have been less likely to occur using the present protocol. On the other hand, nonmonotonic coding might reflect the existence of tuning curves that reveal the general mechanism of information processing by local cortical networks. Such a mechanism, observed for reward processing in other frontal areas (Padoa-Schioppa \& Assad, 2006; Wallis \& Miller, 2003), could also be applied to the ACC. Additional studies will be needed to further investigate the encoding properties of ACC neurons and the respective influences of context and reward schedule.

Importantly, our data show that within a particular context (block of trials, range of available rewards), values are encoded relative to each other. The relative encoding of reward values reveals adaptive properties of neural coding in ACC and suggests that relative encoding of rewards can be adjusted to any learned context. Adaptive coding is not specific to the ACC. Other brain structures (mesencephalic dopaminergic neurons, OFC, and striatum) encode relative reward values of expected or obtained rewards within a scale (Cromwell \& Schultz, 2003; Tobler et al., 2005; Tremblay \& Schultz, 1999). The adaptation of neural encoding is an important mechanism for the reward system to encode an infinite number of possible reward values in specific or changing environments.

\section{Violation of Reward Expectations}

In situations in which reward-size expectations were violated by an incongruent event (expect task), the ACC showed activities that were signaling a gain or loss in re- 
A

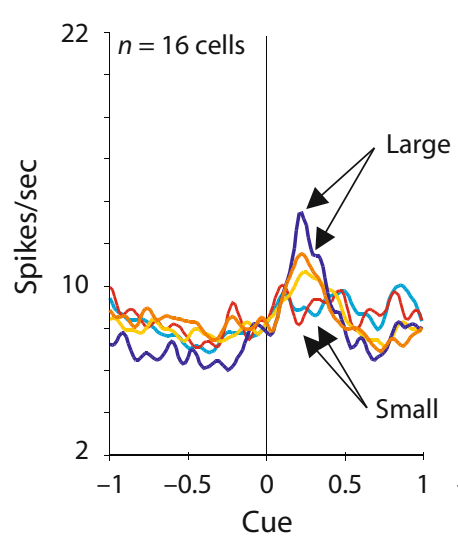

C

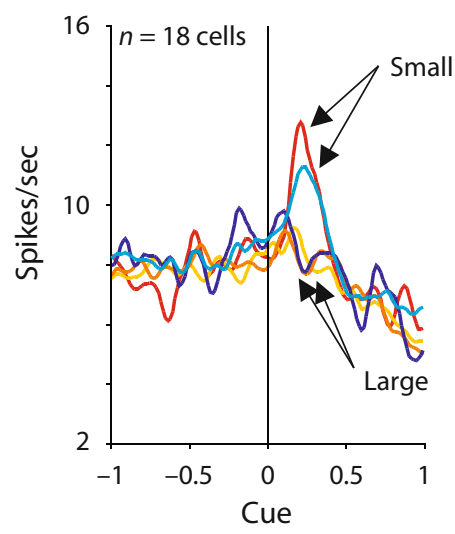

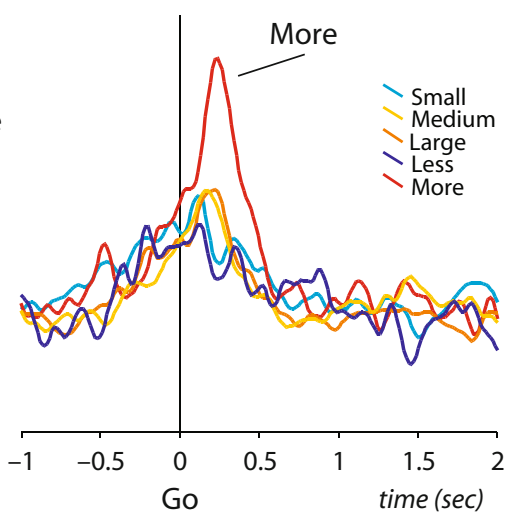

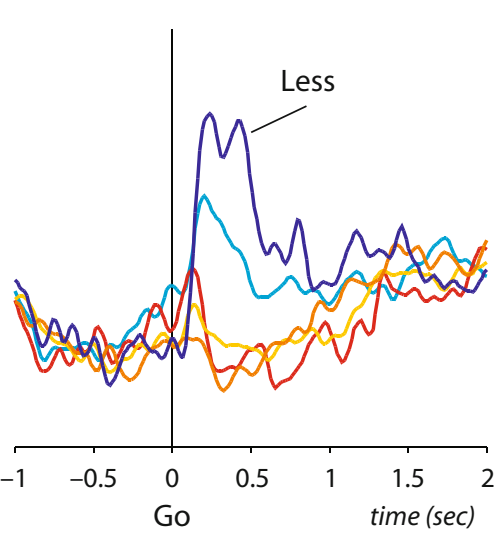

B
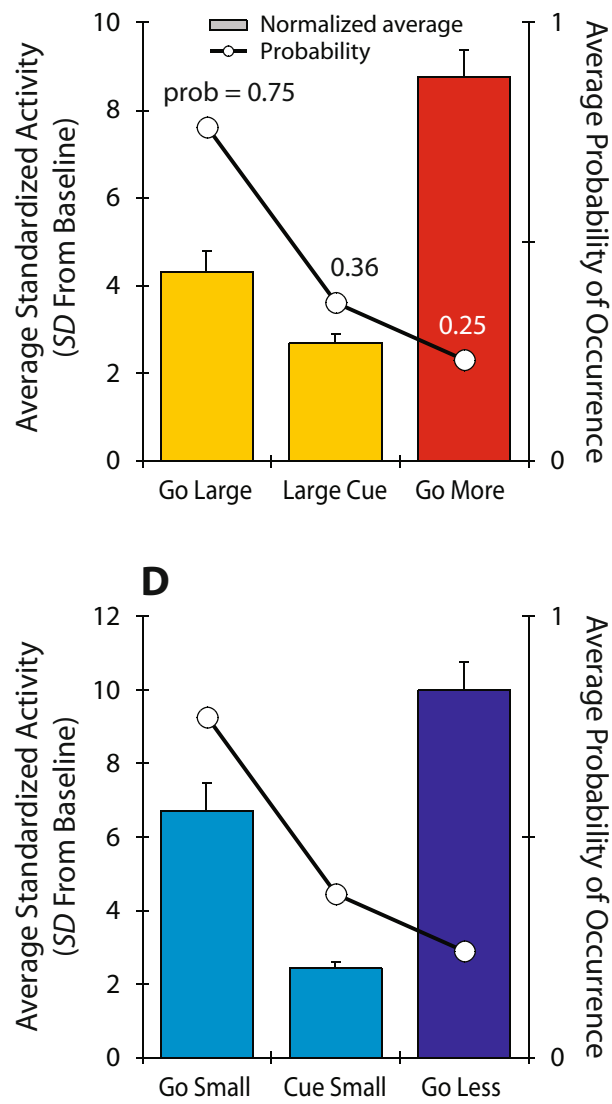

Figure 7. Population activity for two groups of cells in the expect task. (A) Average population activity for cells showing significant activity and maximum average at the go signal in small-more trials. Average activity obtained for each condition is aligned on cue and go separately. Colors indicate conditions as in Figure 6. Note that there is congruence between reward size preference at the cue and at the go signal, and that there is a strong magnification effect combined with a reward size effect at the go signal when the go item indicates an unexpected reward size. (B) Bar graph of average standardized activity ([activity-mean baseline]/SD baseline; see Method) for the different events ranked according to their average predictability in trials. The predictability (probability) is indicated on the superimposed plot. The figure shows that response to events is not following the probability. (C) Average activity for cells showing significant activity and maximum average at the go signal in Large-less trials. (D) same as in (C), but for the less population.

ward. This finding adds to our previous report of ACC error-related activities that are modulated by expectations (Amiez et al., 2005). In human ERP experiments, a midfrontal negativity called medial frontal negativity (MFN) was found to reflect gain or losses (Gehring \& Willoughby, 2002). The origin of the MFN is supposed to be in the ACC. The present study obtained the first unit activity data that directly support this hypothesis. Unit recordings confirm that ACC not only detects unexpected events but also evaluates them in terms of gain or loss in reward. At the population level, our data reveal that ACC encodes and discriminates both gains and losses (i.e., discrepancies in terms of rewards). The fact that ACC cells encode specifically gains or losses suggests that the output of the area concerns different neural populations in target structures and thus possibly induces different responses-for instance, in terms of adaptation.

Our experiment shows that this computation can take place in ACC even before any reward is delivered, by com- parison of the value associated to a reward-predicting cue with the current expectation. This result is comparable to the MFN observed during a "slot-machine" task, in which the evaluation of outcomes was not contingent on any preceding choice or action (Donkers, Nieuwenhuis, \& Boxtel, 2005). Importantly, the expect task involves a motor response that arises just after the evaluative signals; thus, any activity we recorded here can be interpreted in terms of action valuation.

\section{Conclusions}

Major theoretical positions regarding ACC function relate to error detection, conflict monitoring, and outcomeaction association. They converge on the notion that ACC is detecting and evaluating unexpected events that relate to goal achievement and is using this evaluation to trigger adaptive mechanisms.

Our data show first that ACC activity encodes expected reward values, gains, and losses. Thus, our data support a 
role of ACC in value estimation and computation of prediction errors, which are two key elements for reinforcement learning. One important result is that the encoding of values is relative. In other words, reward values are encoded in relation to alternative rewards (the context) and not on an absolute scale. Coding relative values is of primary importance for a structure involved in behavioral adaptation and internally guided decision making.

\section{AUTHOR NOTE}

Thanks to H. Kennedy for helpful comments and language editing. As part of our research on neurobiology of executive functions, these experiments benefited from funds from Inserm, Région Rhône Alpes, Agence National de la Recherche, Fyssen, and NRJ foundations. E.P. and J.P.J. are employed by the CNRS. J.S. is funded by the Ministère de l'Education et Recherche and the Fondation pour la Recherche Médicale. M.R. is funded by the Ministère de l'Education et Recherche, J.V. by the Région Rhône Alpes, and R.Q. by the Facultad de Medicina, Universidad de Valparaíso, proyecto MECESUP UVA-106, and by the Fondation pour la Recherche Médicale. Address correspondence to J. Sallet, Inserm, U846, Stem Cell and Brain Research Institute, 18 Avenue Doyen Lepine, 69500 Bron, France (e-mail: sallet@lyon.inserm.fr).

\section{REFERENCES}

Amiez, C., Joseph, J. P., \& ProcyK, E. (2005). Anterior cingulate errorrelated activity is modulated by predicted reward. European Journal of Neuroscience, 21, 3447-3452.

Amiez, C., Joseph, J. P., \& ProcyK, E. (2006). Reward encoding in the monkey anterior cingulate cortex. Cerebral Cortex, 16, 1040-1055.

Botvinick, M. M., Cohen, J. D., \& Carter, C. S. (2004). Conflict monitoring and anterior cingulate cortex: An update. Trends in Cognitive Sciences, 8, 539-546.

Cromwell, H. C., \& Schultz, W. (2003). Effects of expectations for different reward magnitudes on neuronal activity in primate striatum. Journal of Neurophysiology, 89, 2823-2838.

Donkers, F. C. L., Nieuwenhuis, S., \& van Boxtel, G. J. M. (2005) Mediofrontal negativities in the absence of responding. Cognitive Brain Research, 25, 777-787.

Gehring, W. J., \& Willoughby, A. R. (2002). The medial frontal cortex and the rapid processing of monetary gains and losses. Science, 295, 2279-2282.

Holroyd, C. B., \& Coles, M. G. (2002). The neural basis of human error processing: Reinforcement learning, dopamine, and the errorrelated negativity. Psychological Review, 109, 679-709.

Ito, S., Stuphorn, V., Brown, J. W., \& Schall, J. D. (2003). Performance monitoring by the anterior cingulate cortex during saccade countermanding. Science, 302, 120-122.

Kennerley, S. W., Walton, M. E., Behrens, T. E., Buckley, M. J., \& RushWORTH, M. F. (2006). Optimal decision making and the anterior cingulate cortex. Nature Neuroscience, 9, 940-947.

Kerns, J. G., Cohen, J. D., MacDonald, A. W., III, Cho, R. Y.,
Stenger, V. A., \& CARTer, C. S. (2004). Anterior cingulate conflict monitoring and adjustments in control. Science, 303, 1023-1026.

LEE, D., \& SEO, H. (2007). Mechanisms of reinforcement learning and decision making in the primate dorsolateral prefrontal cortex. In B. W. Balleine, K. Doya, J. O. Doherty, \& M. Sakagami (Eds.) Reward and decision making in corticobasal ganglia networks (Annals of the New York Academy of Sciences, Vol. 1104, pp. 108-122). New York: New York Academy of Sciences.

Matsumoto, M., Matsumoto, K., Abe, H., \& Tanaka, K. (2007). Medial prefrontal cell activity signaling prediction errors of action values. Nature Neuroscience, 10, 647-656.

Padoa-Schioppa, C., \& Assad, J. A. (2006). Neurons in the orbitofrontal cortex encode economic value. Nature, 441, 223-226.

Procyk, E., Amiez, C., Quilodran, R., \& JosePh, J. P. (in press). Modulations of prefrontal activity related to cognitive control and performance monitoring. In P. Haggard, Y. Rosetti, \& M. Kawato (Eds.), Attention and performance XXII: Sensorimotor foundations of higher cognition. New York: Oxford University Press.

ProcyK, E., TanaKa, Y. L., \& JosePh, J. P. (2000). Anterior cingulate activity during routine and non-routine sequential behaviors in macaques. Nature Neuroscience, 3, 502-508.

Ridderinkhof, K. R., Ullsperger, M., Crone, E. A., \& NieuwenHUIS, S. (2004). The role of the medial frontal cortex in cognitive control. Science, 306, 443-447.

Rushworth, M. F. S., Behrens, T. E. J., Rudebeck, P. H., \& Walton, M. E. (2007). Contrasting roles for cingulate and orbitofrontal cortex in decisions and social behaviour. Trends in Cognitive Sciences, 11, $168-176$

Rushworth, M. F. S., Walton, M. E., Kennerley, S. W., \& BannerMAN, D. M. (2004). Action sets and decisions in the medial frontal cortex. Trends in Cognitive Sciences, 8, 410-417.

Schultz, W. (2000). Multiple reward signals in the brain. Nature Reviews Neuroscience, 1, 199-207.

Shima, K., Aya, K., Mushiake, H., Inase, M., Aizawa, H., \& Tanji, J. (1991). Two movement-related foci in the primate cingulate cortex observed in signal-triggered and self-paced forelimb movements. Journal of Neurophysiology, 65, 188-202.

Shima, K., \& TANJI, J. (1998). Role for cingulate motor area cells in voluntary movement selection based on reward. Science, 282, 1335-1338.

Tobler, P. N., Fiorillo, C. D., \& Schultz, W. (2005). Adaptive coding of reward value by dopamine neurons. Science, 307, 1642-1645.

Tremblay, L., \& Schultz, W. (1999). Relative reward preference in primate orbitofrontal cortex. Nature, 398, 704-708.

WALLIS, J. D., \& MilLER, E. K. (2003). Neuronal activity in primate dorsolateral and orbital prefrontal cortex during performance of a reward preference task. European Journal of Neuroscience, 18, 2069-2081.

Walton, M. E., Devlin, J. T., \& Rushworth, M. F. (2004). Interactions between decision making and performance monitoring within prefrontal cortex. Nature Neuroscience, 7, 1259-1265.

(Manuscript received February 3, 2007; revision accepted for publication August 31, 2007.) 